\title{
Diagnostic Utility of Ki-67 for Histopathological Typing of Conventional Ameloblastoma, Proliferative Ameloblastoma and Ameloblastic Carcinoma - A Retrospective Study
}

\author{
Mithra S. ${ }^{1}$, Archana Santhanam ${ }^{2}$, Herald J. Sherlin ${ }^{3}$, Gifrina Jayaraj ${ }^{4}$, Don K.R. ${ }^{5}$ \\ 1, 2,3,4,5 Department of Oral and Maxillofacial Pathology, Saveetha Dental College and Hospitals, \\ Saveetha Institute of Medical and Technical Sciences, Chennai, Tamil Nadu, India.
}

\section{ABSTRACT}

\section{BACKGROUND}

Ameloblastoma is a rare, benign odontogenic neoplasm which is locally aggressive and mostly present with a painless swelling. The enigma about the diagnosis of proliferative ameloblastoma and ameloblastic carcinoma is still a debate because the diagnostic criteria is not standardized or quantified which has a direct correlation on its biological behaviour and prognosis. Despite numerous studies, correlation between the histological patterns of ameloblastoma and tumour behaviour has not been consistently established. The present study was done to compare the expression levels of Ki-67 between conventional ameloblastoma, proliferative ameloblastoma and ameloblastic carcinoma and to assess the usefulness of these markers for diagnostic differentiation.

\section{METHODS}

A retrospective study of total of 18 cases of ameloblastoma were retrieved from the archives of Department of Oral and Maxillofacial Pathology, Saveetha Dental College from 2012 till 2019, which included conventional ameloblastoma, proliferative ameloblastoma and ameloblastic carcinoma. Immunohistochemical (IHC) analysis was done using the marker Ki-67 and labelling index were determined for the same.

\section{RESULTS}

The results of the current study showed that the cellular proliferative activity assessed using Ki-67 in follicular ameloblastoma was (55\%), 4 cases of plexiform ameloblastoma ( $22 \%$ ), $17 \%$ of proliferative ameloblastoma and $6 \%$ of ameloblastic carcinoma showed negative expression.

\section{CONCLUSIONS}

Immunophenotyping using the marker Ki-67 may be a useful tool for histological typing of ameloblastoma.
Corresponding Author: Dr. Mithra S,

Department of Oral and Maxillofacial Pathology, Saveetha Dental College and Hospitals, Saveetha Institute of Medical and Technical Sciences, Chennai, Tamil Nadu, India.,

E-mail:mithraswomappan@grnail.com

DOI: $10.14260 / \mathrm{jemds} / 2021 / 531$

How to Cite This Article:

Mithra S, Santhanam A, Sherlin HJ, et al. Diagnostic utility of KI

-67 for histopathological typing of conventional ameloblastoma, proliferative ameloblastoma and ameloblastic carcinoma - a retrospective study. J Evolution Med Dent Sci 2021;10(32):25922596, DOI: 10.14260/jemds/2021/531

Submission 03-11-2020,

Peer Review 08-06-2021,

Acceptance 14-06-2021,

Published 09-08-2021.

Copyright (C) 2021 Mithra S. et al. This is an open access article distributed under Creative Commons Attribution License [Attribution 4.0 International (CC BY 4.0)]

\section{KEY WORDS}

Ameloblastoma, Ki-67, IHC, Immunophenotyping 


\section{BACKGROUND}

Group of lesions that arise from tooth forming tissues or their remnants are the odontogenic cysts and tumours which originate from odontogenic epithelium or ectomesenchyme with various degrees of inductive tissue interactions. ${ }^{1}$ Different odontogenic cysts and tumours have variable clinical and biological behaviors. ${ }^{2}$ Ameloblastoma is a local, aggressive, benign odontogenic neoplasm that attained greater consideration in both medical and dental literature from the 19th century. According to the World Health Organization (WHO) classification, the clinical types of ameloblastoma are: conventional, unicystic, extraosseous / peripheral, and metastasizing ameloblastoma. ${ }^{3}$ Histologically, ameloblastoma is classified as follicular, plexiform, acanthomatous, granular, desmoplastic, and basal. With the radiographic features, it has been classified as unicystic or multicystic. ${ }^{4}$ Among all the types, about $86 \%$ of the cases are being reported as multicystic or solid type. There are also 3 subtypes for the unicystic type which are luminal, intraluminal and mural. ${ }^{5}$ Ameloblastomas are the second most common odontogenic tumours following the odontome. Ameloblastoma usually presents as a painless, slow growing, non-metastatic, locally invasive swelling that histologically resembles the enamel organ of developing teeth but doesn't form dental hard tissues.

Depending on various factors like the size, histological variant, anatomical location and involvement, the treatment is decided. ${ }^{6}$ There are about $1 \%$ of the cases that have been reported for malignant transformation. These malignant types of ameloblastoma may arise or may even transform from an already existing ameloblastoma. Spread from lungs, cervical lymph nodes are reported as the commonest sites of metastasis. ${ }^{7}$

Despite numerous studies, correlation between the histological patterns of ameloblastoma and tumour behaviour has not been consistently established. The mechanism of oncogenesis, tumour progression of ameloblastoma are still largely unknown. The enigma about the diagnosis of proliferative ameloblastoma and ameloblastic carcinoma is still a debate because the diagnostic criteria are not standardized or quantified which has a direct correlation on its biological behaviour and prognosis. ${ }^{8}$

Determination of epithelial proliferative activity plays a crucial role in determining the biological behaviour of the tumour, tumour classification and monitoring treatment response and relapse. ${ }^{8} \mathrm{Ki}-67$ is one of the most commonly used proliferative IHC markers to evaluate proliferative activity and biologic behaviour of most pathologic lesions. Ki-67 is a nonhistone nuclear protein having correlations with cell growth. The expression of Ki-67 usually has alterations throughout the cell cycle with various expression levels in mid G1, increase in $\mathrm{S}$ and $\mathrm{G} 2$ with a peak in mitosis (M). The absence of this marker in the G0 phase helps to determine the growth fraction of a cell population. Ki-67 is detectable in the nucleus of proliferating cells. Ki-67 is one clear cut marker for proliferating cells which are mostly found during $\mathrm{S}$ phase. ${ }^{9} \mathrm{Ki}-67$ expression in ameloblastoma is associated with a more invasive pattern of growth with increased rate of recurrence.

The proliferation of cells is one necessary step that takes place in all living organisms as it has a major part in maintaining the homeostasis of the tissues and growth of the cells. ${ }^{10}$ When it comes to neoplasms, the proliferative activity is completely altered compared to the normal process. ${ }^{8}$ At this time, the necessity for evaluating the cell proliferative activity through immunohistochemistry became one important technique to provide beneficial understanding upon the nature of various tumors. ${ }^{9}$ The incorporation of various immunologic research methods to histopathology has helped to emerge a notable increase in the microscopic diagnosis of neoplasms. Despite the fact that tissue sections stained with haematoxylin and eosin stand first when it comes to histological analysis, immunohistochemistry has become an effective tool in the armamentarium of the pathologists. ${ }^{11}$ Immunohistochemistry is one technique which helps in recognizing the cellular / tissue components by antigenantibody interactions. Immunohistochemistry mainly focuses on markers of specific cells and tumour type that guides in the diagnosis of the specific tumour. ${ }^{12}$

The main objective of this study was to determine the immunohistochemical expression of Ki-67 in conventional ameloblastoma, proliferative ameloblastoma and ameloblastic carcinoma, and to perform the histological typing of the ameloblastoma based on the expression of Ki-67.

\section{METHODS}

This is a retrospective study. A total of 18 cases histologically diagnosed as ameloblastoma were selected from 2012 to 2019. Based on the histopathology reports, the cases were classified into different types and selected accordingly. Different histological types of ameloblastoma included in the study were conventional follicular ${ }^{10}$, conventional plexiform ${ }^{4}$, proliferative ameloblastoma ${ }^{3}$ and ameloblastic carcinoma. ${ }^{1}$ Epidemiological, clinical data and outcome were collected by retrospective review of the patient's charts from the department of oral \& maxillofacial surgery of the same hospital.

Histological slides were reviewed and slides comprising the largest amount of tumour tissue and not containing any bone fragments were selected in each case and the corresponding paraffin embedded blocks were retrieved from the archives of the Department of Oral and Maxillofacial Pathology, Saveetha Dental College, Chennai.

\section{Immunohistochemistry}

$3 \mu \mathrm{m}$ sections were cut from formalin-fixed paraffinembedded blocks mounted on gelatin-coated slides. Then sections were deparaffinized in xylene for 10 minutes \& followed by dehydration in $100 \%$ alcohol for 5 minutes and rinsed in distilled water. Following which heat mediated antigen retrieval with Tris - ethylene diamine tetra acetic acid (EDTA) buffer solution of $9.0 \mathrm{pH}$ was done in a pressure cooker for 5 minutes. Depressurize the pressure cooker to $37 \mathrm{c}$ under running tap water. Endogenous peroxidase was blocked for 30 minutes. Sections were incubated with primary antibody, Ki67 (Dako, FLEX Monoclonal mouse anti-human Ki-67 antigen clone, Denmark) for 1 hour at room temperature. Sections were washed and incubated with secondary antibody (Polyexcel HRP) for 30 minutes at room temperature. 
Detection was performed using polyexcel HRP/DAB detection system (Pathnsitu, conjugated by goat anti mouse/rabbit IgG, USA). The sections were then counterstained with Mayer's haematoxylin and were then dehydrated and mounted using dibutyl phthalate in xylene mountant. Negative and positive controls were used in each run.

\section{Scoring Criteria}

Positive reactivity was determined by the existence of the brown coloured reactions at the site of target antigen. Ki-67 labelling index was done by calculating the number of positive tumour cells/total number of tumour cells and expressed as a percentage. The scores are tabulated in table 1.

\section{Statistical Analysis}

All the results were tabulated and assessed for statistical analysis using Statistical Package for Social Sciences (SPSS) (IBM SPSS Statistics for Mac Version 20.0). Difference between mean values was considered statistically significant when $P$ value was $\leq 0.05$.

\section{RESULTS}

A total of 18 histopathologically diagnosed ameloblastoma cases were included in this study. The selected cases were analysed for immunohistochemistry staining for the expression of Ki-67. Table 2 shows the patients characteristics. In the current study, the male : female ratio was $1: 1$. Age distribution ranged from 12 to 67 years, with a mean age of 34.71 years. The specimens comprised of 10 cases of follicular ameloblastoma (55\%), 4 cases of plexiform ameloblastoma (22\%), $17 \%$ of proliferative ameloblastoma and $6 \%$ of ameloblastic carcinoma.

\begin{tabular}{|ccc|}
\hline Score & Proportionality Index & Intensity of Staining \\
0 & No labelling & Negative \\
1 & 0 to $7 \%$ of tumour cells are labelled & Mild \\
2 & 8 to $15 \%$ of tumour cells are labelled & Moderate \\
3 & $>15 \%$ of tumour cells are labelled & Severe \\
\hline Table & 1. Scoring Criteria for Evaluation of Expression of $\mathbf{K i}-67$ \\
\hline
\end{tabular}

\begin{tabular}{|ccc|}
\hline Factor & Group & Total Sample (N \%) \\
Gender & Male & $9(50 \%)$ \\
& Female & $9(50 \%)$ \\
Age & $<50$ & $14(78 \%)$ \\
& $>$ & $4(22 \%)$ \\
Anatomical location & Anterior & 0 \\
Site & Posterior & $15(83 \%)$ \\
& Mandible & $18(100 \%)$ \\
Histopathological type & Maxilla & 0 \\
& Conventional follicular & $10(55 \%)$ \\
& Conventional plexiform & $4(22 \%)$ \\
Recurrence & Proliferative & $3(17 \%)$ \\
& Ameloblastic carcinoma & $1(6 \%)$ \\
& Yes & $1(6 \%)$ \\
& No & $17(94 \%)$ \\
\hline
\end{tabular}

\begin{tabular}{|ccc|}
\hline $\begin{array}{c}\text { Histopathological Type } \\
\text { (n=18) }\end{array}$ & Ki67 Expression & Level of Expression \\
Conventional follicular & Negative & NA \\
Conventional plexiform & Negative & NA \\
Proliferative & Negative & NA \\
\hline Ameloblastic carcinoma & Negative & NA \\
\hline Table 3. Expression of $\mathbf{K}$ - $\mathbf{6 7}$ According to the \\
Histopathological Characteristics of Ameloblastoma \\
\hline
\end{tabular}

Tumours were predominantly located in the posterior regions of the mandible. Recurrence of the ameloblastoma occurred in 1 case out of 18 and was seen in male. The results showed that Ki-67 showed negative expression irrespective of different types of ameloblastoma.

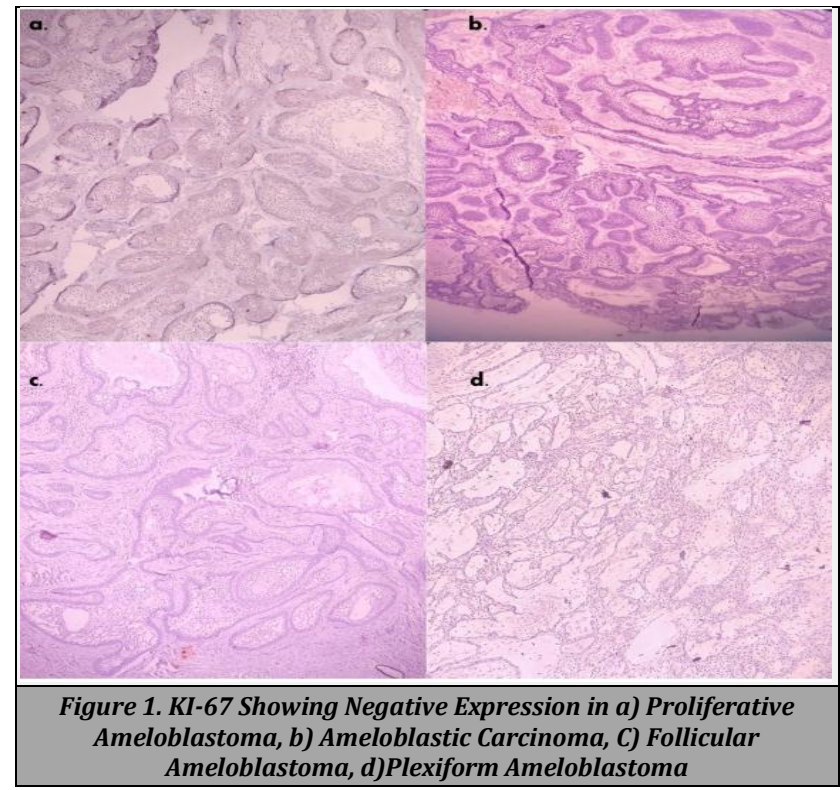

\section{DISCUSSION}

Immunohistochemistry is a useful diagnostic tool for both monoclonal and polyclonal antibodies to discover the definite antigen within the tissue section wherein it is widely used in the diagnosis of cancers. ${ }^{13}$ IHC plays a very crucial role in the diagnosis of many diseases as well. It has now become an essential part of diagnosis most widely used in medical research and clinical diagnostics. ${ }^{14}$ Ameloblastoma is a benign, local, aggressive tumour with high rate of recurrence and has a probability of malignant transformation. ${ }^{15}$

The clinical course in patients with ameloblastoma is difficult to predict and also there is no clear evidence that histologic features of ameloblastoma determine the degree of invasiveness, recurrence rate or malignant transformation. Therefore, there is a need for a predictive marker for clinical behaviour in ameloblastoma. The proliferative activity of tumour cells has been found to be an indicator of aggressiveness and aids in determining the prognosis of many tumours. The use of Ki- 67 before surgical excision of the ameloblastoma may be an important step in the treatment plan to minimize the recurrence rate.

The results of the current study showed that cellular proliferative activity assessed using Ki-67 in recurrent, nonrecurrent cases of ameloblastoma and ameloblastic carcinoma showed negative expression. This could be due to the small sample size of our study. The cell proliferative activity in ameloblastoma and ameloblastic carcinoma shows positive expression for Ki-67 whereas non proliferating cells that have the tendency to hark back to cell division cycle are expressed as negative for $\mathrm{Ki}-67.16$

With regards to ameloblastic carcinoma, due to the rarity of this tumour, this study could include only one case. Chae et al. in his study proposed that there was no significant or clear 
correlation between the proliferative activity and the clinical values. ${ }^{17}$ At the same time Carreon et al. observed a highest proliferative index in mandible with tumours measuring more than $5 \mathrm{~cm}$ in female patients with no recurrence. ${ }^{18}$ In this study, no significant correlations between clinical characteristics, recurrence, and protein expression were observed. Florescu et al. found a considerable variation of Ki67 expression amidst central and peripheral cells which were considered to be zones of proliferation in an array of 14 ameloblastoma cases ${ }^{19}$ Also in another study it was observed that few cells in the stellate reticulum like cells had positive expression for Ki-67 along with predominant peripheral cell positivity. ${ }^{20}$ Wherein a study by Ahlem et al. observed that Ki67 expression was considerably more in recurrent tumors. ${ }^{21}$ Another study by A. Hegab et al. also stated that there was a significant relation between the labelling index of Ki-67 and the recurrence of the disease, which was found to be higher in plexiform type followed by follicular type. He also in his study stated that there were variations in the cellular proliferating activity in different types of ameloblastoma and he also observed that statistically there was higher significance of Ki67 in recurrent ameloblastoma than in non-recurrent ameloblastomas. ${ }^{22}$ There are also several studies which have shown that the Ki-67 labelling index is prognostically relevant. 23,24

\section{CONCLUSIONS}

In future, studies assessing Ki-67 expression in ameloblastoma and ameloblastic carcinoma with a longer follow-up period and with higher rates of recurrence is necessary. The current study suggests that there is no direct association between cell proliferation and clinical parameters. The significant expression of Ki-67 can be used as a prognostic marker as it is more specific for proliferating cells.

Data sharing statement provided by the authors is available with the full text of this article at jemds.com.

Financial or other competing interests: None.

Disclosure forms provided by the authors are available with the full text of this article at jemds.com.

The authors would like to acknowledge the help and support rendered by the Department of Oral Pathology of Saveetha Dental College and Hospitals and the management for their constant assistance with the research.

\section{REFERENCES}

[1] Fernandes AM, Duarte ECB, Pimenta FJGS, et al. Odontogenic tumors: a study of 340 cases in a Brazilian population. J Oral Pathol Med 2005;34(10):583-7.

[2] Reichart PA, Reichart P, Philipsen HP. Odontogenic Tumors and Allied Lesions. London: Quintessence 2004:43-59.

[3] Thompson LDR. Update from the $4^{\text {th }}$ edition of the world health organization classification of head and neck tumours: tumours of the ear. Head Neck Pathol 2017;11(1):78-87.

[4] Escande C, Chaine A Menard P, et al. A treatment algorythmn for adult meloblastomas according to the
Pitié-Salpêtrière Hospital experience. J Craniomaxillofac Surg 2009;37(7):363-9.

[5] Sampson DE, Pogrel MA. Management of mandibular ameloblastoma: the clinical basis for a treatment algorithm. J Oral Maxillofac Surg 1999;57(9):1074-7.

[6] Feinberg SE, Steinberg B. Surgical management of ameloblastoma. Oral Surg Oral Med Oral Pathol Oral Radiol Endod 1996;81(4):383-8.

[7] Bachmann AM, Linfesty RL. Ameloblastoma, solid/multicystic type. Head Neck Pathol 2009;3(4):3079.

[8] Tumuluri V, Thomas GA, Fraser IS. Analysis of the KI-67 antigen at the invasive tumour front of human oral squamous cell carcinoma. J Oral Pathol Med 2002;31(10):598-604.

[9] Bologna-Molina R, Mosqueda-Taylor A, Molina-Frechero $\mathrm{N}$, et al. Comparison of the value of PCNA and Ki-67 as markers of cell proliferation in ameloblastic tumors. Med Oral Patol Oral Cir Bucal 2013;18(2):e174-9.

[10] Van Diest PJ, Brugal G, Baak JPA. Proliferation markers in tumours: interpretation and clinical value. J Clin Pathol 1998;51(10):716-24.

[11] Jordan RCK, Daniels TE, Greenspan JS, et al. Advanced diagnostic methods in oral and maxillofacial pathology. Part II: immunohistochemical and immunofluorescent methods. Oral Surg Oral Med Oral Pathol Oral Radiol Endod 2002;93(1):56-74.

[12] Kabiraj A, Gupta J, Khaitan T, et al. Principle and techniques of immunohistochemistry-a review. Int J Biol Med Res 2015;6(3):5204-10.

[13] Duraiyan J, Govindarajan R, Kaliyappan K, et al. Applications of immunohistochemistry. J Pharm Bioallied Sci 2012;4(Suppl 2):S307-9.

[14]Ajura AJ, Sumairi I, Lau SH. The use of immunohistochemistry in an oral pathology laboratory. Malays J Pathol 2007;29(2):101-5.

[15] Sivapathasundharam B, Rajendran R. Shafer'S Textbook of Oral Pathology. $6^{\text {th }}$ edn. Elsevier India 2009: p. 963.

[16] Endl E, Kausch I, Baack M, et al. The expression of Ki-67, MCM3 and p27 defines distinct subsets of proliferating, resting and differentiated cells. J Pathol 2001;195(4):45762.

[17] Chae MP, Smoll NR, Hunter-Smith DJ, et al. Establishing the natural history and growth rate of ameloblastoma with implications for management: systematic review and meta-analysis. PLoS One 2015;10(2):e0117241.

[18] Carreón-Burciaga RG, González-González R, MolinaFrechero N, et al. Immunoexpression of Ki-67, MCM2 and MCM3 in ameloblastoma and ameloblastic carcinoma and their correlations with clinical and histopathological patterns. Dis Markers 2015;2015:683087.

[19] Florescu A, Simionescu C, Ciurea R, et al. P53, Bcl-2 and Ki67 immunoexpression in follicular solid ameloblastomas. Rom J Morphol 2012 J;53(1):105-9.

[20] Tekkeşın MS, Mutlu S, Olgaç V. Expressions of bax, bcl-2 and Ki-67 in odontogenic keratocysts (Keratocystic Odontogenic Tumor) in comparison with ameloblastomas and radicular cysts. Turk Patoloji Derg 2012;28(1):49-55.

[21] Ahlem B, Wided A, Amani L, et al. Study of Ki67 and CD10 expression as predictive factors of recurrence of 
ameloblastoma. Eur Ann Otorhinolaryngol Head Neck Dis 2015;132(5):275-9.

[22] Hegab AF, Shuman M, Abd El-Akher M, et al. Ki-67 immunohistochemical expression in mandibular ameloblastoma: a prognostic indicator for local recurrence. Open Journal of Stomatology 2013;3(9):5206.
[23] Wintzer HO, Zipfel I, Schulte-Mönting J, et al. Ki-67 immunostaining in human breast tumors and its relationship to prognosis. Cancer 1991;67(2):421-8.

[24] Veronese SM, Gambacorta M, Gottardi 0, et al. Proliferation index as a prognostic marker in breast cancer. Cancer 1993;71(12):3926-31. 